\title{
DER DEUTSCHE WEG ZUM ÉLYSÉE-VERTRAG
}

Der deutsche Weg zum Élysée-Vertrag ist von der Wissenschaft schon hell ausgeleuchtet. Von den französischen Beleuchtungsspezialisten nenne ich nur die Historiker Jacques Bariéty ${ }^{1}$ und Georges-Henri Soutou, ${ }^{2}$ von den deutschen Ulrich Lappenküper, der unter gleichzeitiger, akribischer Auswertung eines fast lückenlosen französischen und deutschen Quellenbestands eine, möchte ich sagen, weitgehend definitive Darstellung publiziert hat. ${ }^{3}$ Sie analysieren in ihren Studien die vielschichtige Entwicklung, die schließlich zur Unterschrift am 22. Januar 1963 führte, so daß ich mich nicht mit einer Wegeskizze aufhalten möchte, ${ }^{4}$ sondern vielmehr den Gesamtvorgang in eine etwas umfassendere Perspektive rücken und, davon ausgehend, einige kritische Punkte ansprechen will, die mir wichtig erscheinen.

1 Vgl. Jacques BARIÉTY, Die Rolle der persönlichen Beziehungen zwischen Bundeskanzler Adenauer und General de Gaulle für die deutsch-französische Politik zwischen 1958 und 1963, in: Hans-Peter ScHWARZ (Hg.), Adenauer und Frankreich, Bonn 1985 (Rhöndorfer Gespräche, 7), S. 12-27; DERS., De Gaulle, Adenauer et la genèse du traité de l'Élysée du 22 janvier 1963, in: Institut Charles de Gaulle (Hg.), De Gaulle et son siècle, Bd. 5 : L'Europe, Paris 1992, S. 352-364; DERS., Les entretiens de Gaulle - Adenauer de juillet 1960 à Rambouillet: Prélude au plan Fouchet et au traité de l'Élysée, in: Revue d'Allemagne et des pays de langue allemande, 29 (1997) 2, S. 167-175.

2 Vgl. Georges-Henri Soutou, Le général de Gaulle et le Plan Fouchet, in: Institut Charles de Gaulle (Hg.), De Gaulle en son siècle, Bd. S: L'Europe, Paris 1992, S. 126-143; DERS., L'alliance incertaine. Les rapports politico-stratégiques franco-allemands, 1954 1996, Paris 1996.

3 Vgl. Ulrich LAPPENKÜPER, Die deutsch-französischen Beziehungen 1949-1963. Von der "Erbfeindschaft" zur "Entente élémentaire «, München 2001. Hingewiesen sei hier auch auf Ansbert BAUMANN, Die organisierte Zusammenarbeit. Die deutsch-französischen Beziehungen am Vorabend des Élysée-Vertrags (1958-1962), Ludwigsburg 2002 (dfi compact, 1).

4 Hier sei verwiesen auf Hans-Peter SCHWARZ, Le président de Gaulle, le chancelier fédéral Adenauer et la genèse du traité de l'Élysée, in: Institut Charles de Gaulle (Hg.), De Gaulle et son siècle, Bd. 5: L'Europe, Paris 1992, S. 364-373; DERS., Präsident de Gaulle, Bundeskanzler Adenauer und die Entstehung des Élysée-Vertrages, in: Karl Dietrich BRACHER u.a. (Hg.), Deutschland zwischen Krieg und Frieden: Beiträge zur Politik und Kultur im 20. Jahrhundert (Festschrift für Hans-Adolf Jacobsen), Düsseldorf 1991, S. 212-220; DERS., Erbfreundschaft. Adenauer und Frankreich - Amitié héréditaire. Adenauer et la France, Bonn, Berlin 1992. 
Zuerst zur weiteren Perspektive. Hier ist auf ein bemerkenswertes Paradox aufmerksam zu machen. Wie wir alle wissen und, so denke ich, auch lebhaft begrüßen, hat der Élysée-Vertrag eine ganz erstaunliche Langzeitwirkung entfaltet. Vieles hat dazu beigetragen: die vertraglich normierte Konsultationspflicht, die Existenz gemeinsamer Gegner, gegen die man sich rüsten, und gemeinsamer Freunde, die man sich doch etwas vom Leibe halten möchte, überhaupt eine breite Palette gemeinsamer Interessen, aber nicht zuletzt auch historische Zufälle. $\mathrm{Zu}$ letzteren zählt die Tatsache, daß relativ viele der politischen Spitzenchargen auf beiden Seiten vergleichsweise gut miteinander konnten - vergleichsweise gut, denn das ist in der von Dauerstreit, Mißtrauen und Profilierungszwängen erfüllten Welt der Politik schon recht viel.

Über die erstaunliche und alles in allem positive Langzeitwirkung des Élysée-Vertrages reflektieren, das hieße auch, die Rolle von Persönlichkeiten wie Georges Pompidou und Willy Brandt, Valéry Giscard d'Estaing und Helmut Schmidt, François Mitterrand und Helmut Kohl, aber auch Außenminister wie Roland Dumas und Hans-Dietrich Genscher angemessen zu diskutieren. Doch bei den Würdigungen, die zu runden Geburtstagen stets Hochkonjunktur haben, wird häufig die eigenartige Tatsache etwas ins Halbdunkel geschoben, $\mathrm{da} ß$ dieser berühmte Vertrag anfänglich einen ganz peinlichen Fehlstart zu verzeichnen hatte. Damit meine ich nicht blo $B$ die eigentliche Entstehungsgeschichte, das Thema meiner Problemskizze, sondern auch die ersten Jahre seines Funktionierens, genauer gesagt: schlechten Funktionierens zwischen 1963 und 1966, als Bundeskanzler Ludwig Erhard und sein Außenminister Gerhard Schröder ${ }^{5}$ einen kompromißlos atlantischen Kurs steuerten, während sich de Gaulle umgekehrt zusehends im Anti-Amerikanismus verrannte. ${ }^{6}$ Der durch die bundesdeutsche Präambel zum Ratifikationsgesetz, auf die noch zurückzukommen ist, bereits politisch entkernte Vertrag erwies sich in jenen Jahren vorwiegend als großer Zankapfel - Zankapfel zwischen den Bonner »Atlantikern« und »Gaullisten «, ${ }^{7}$ Zankapfel innerhalb der Sechsergemeinschaft, Zankapfel im Verhältnis zu Großbritannien, das den Deutschen damals unerklärlicherweise so heilsnotwendig für den Fortschritt des europäischen Zusammenschlusses erschien wie heute die Osterweiterung der EU, und Zankapfel nicht zuletzt im Verhältnis zu Washington.

5 Vgl. Torsten OPPELLAND, Gerhard Schröder (1910-1989). Politik zwischen Staat, Partei und Konfession, Düsseldorf 2002.

6 Vgl. Klaus HiLdebrand, Der provisorische Staat und das ewige Frankreich: Die deutsch-französischen Beziehungen 1963-1969, in: SCHWARZ (Anm. 1), S. 62-81.

7 Vgl. Klaus HILDEBRAND, »Atlantiker« versus »Gaullisten«: zur Außenpolitik der Bundesrepublik Deutschland während der sechziger Jahre, in: Revue d'Allemagne et des pays de langue allemande, 22 (1990) 4, S. 583-592. 
"Je suis resté vierge ${ }^{8},{ }^{2}$ hat de Gaulle schon bald geklagt, und es war erst die Große Koalition unter Bundeskanzler Kurt-Georg Kiesinger ${ }^{9}$ mit dem für die Bedeutung Frankreichs gleichfalls aufgeschlossenen sozialdemokratischen Bundesaußenminister Willy Brandt, die nachhaltig, wenngleich letztlich ohne durchschlagenden Erfolg, um eine gewisse ,Entjungferung، des Generals bemüht war. Brandt verwendete zur Kennzeichnung des deutsch-französischen Sonderverhältnisses während seiner Kanzlerschaft gern den Begriff »entente élémentaire ${ }^{10}{ }^{10}$ und machte dieses Sonderverhältnis zu einem Kernelement seiner Außenpolitik. Das durch den Vertrag geregelte Verhältnis war damit jedem Parteienstreit enthoben, eben weil es als elementar begriffen wurde." $\mathrm{DaB}$ es ab Anfang der siebziger Jahre bergauf ging, lag aber auch nicht zuletzt an dem Willen des sozialdemokratischen Kanzlers und dem neuen gaullistischen Hausherren im Élysée-Palast, Georges Pompidou, der gewiß kein ausgesprochener Germanophiler war, die den Beziehungen während ihrer Amtszeit ihren mythischen Charakter nahmen. Auch wenn dem französischen Staatspräsidenten ihre elementare Bedeutung bewußt war, zog er die »relations exemplaires « den »relations priviligées« vor. Beide hatten damit eine Arbeitsgrundlage geschaffen, auf der auch ihre Nachfolger aufbauen konnten. ${ }^{12}$

Die eigentliche Paradoxie besteht also in der Tatsache, daß ein durch Fehlstart ziemlich verdorbener, im beiderseitigen Streit ausgeleierter, halbtoter Vertrag, reanimiert wurde und von dann an eine dauerhafte Kraft entfaltete. Darauf vor allem sollten die Historiker künftig ihren Scharfsinn verwenden. Doch das ist nicht mein Thema, und so ist jetzt zu fragen, wie es eigentlich dazu gekommen ist, daß ein Vertrag, der so evidente Vorzüge hatte, die auch schon im Jahr 1963 erkennbar waren, von Anfang an zu so bitterem Streit führte. War das aufgrund der Konstellation unvermeidlich? Sind einfach einige Fehler gemacht worden, denen sich weitere Fehler anschlossen? Ich möchte in dieser Beziehung nur drei Fragen aufwerfen:

8 Vgl. zum Kontext: Hans-Peter SCHWARZ, Adenauer. Der Staatsmann: 1952-1967, Stuttgart 1991, S. 888.

- Vgl. Ansbert BaumanN, Kurt Georg Kiesinger - Ein Freund Frankreichs?, in: Historisch-Politische Mitteilungen. Archiv für Christlich-Demokratische Politik, 10 (2003), S. 225-253.

10 Willy BRANDT, Begegnungen und Einsichten. Die Jahre 1960-1975, Hamburg 1976, S. 341 .

"Vgl. Hans-Peter SCHWARZ, Eine Entente Elémentaire. Das deutsch-französische Verhältnis im 25. Jahr des Élysée-Vertrages. Mit einer Dokumentation von Ingo KolBOOM, Bonn 1988, S. 6ff.

12 Vgl. Andreas WILKENS, Accords et désaccords. La France, l'Ostpolitik et la question allemande 1969-1974, in: Ulrich PFEIL (Hg.), La RDA et l'Occident 1949-1990, Asnières 2000, S. 357-378; DERS., Relance et réalités. Willy Brandt, la politique européenne et les institutions communautaires, in: Marie-Thérèse BiTSCH (Hg.), Le couple France-Allemagne et les institutions européennes. Une postérité pour le Plan Schuman, Brüssel 2001, S. $377-418$. 
1. Wäre alles nicht viel besser gelaufen, wenn Adenauer beim ursprünglichen Konzept eines nicht ratifikationsbedürftigen Protokolls geblieben wäre, statt im allerletzten Moment, völlig unvorbereitet, auf das Konzept eines Vertrages umzuschwenken?

2. Wäre nicht alles viel besser gelaufen, hätte de Gaulle nicht seine berüchtigte Pressekonferenz vom 14. Januar 1963 abgehalten, von der Adenauer bald darauf hinter vorgehaltener Hand selber sagte, sie sei $»$ schrecklich ${ }^{13}$ und sie sei »ein Fehler ${ }^{14}$ gewesen?

3. Hat nicht erst die vom aufgeregten Bonner Parteien-Establishment und von den aufgeregten bundesdeutschen Medien im Verein mit starken äußeren Einflüssen Washingtons und Londons durchgesetzte Präambel den Vertrag für eine Reihe von Jahren zum Zankapfel gemacht?

Man verstehe mich richtig. Ich möchte nicht nachträglich analysieren, ob und wie man es besser hätte anfangen können - derartige nachträgliche Historiker-Klugheiten sind immer von Übel. Ohnehin bleibt zu fragen: Hätte ein bloßes Regierungsabkommen in Form eines Protokolls statt des tatsächlich zustande gekommenen Vertrages die frustierenden ersten drei Jahre überhaupt überstanden und dann eine langfristige Wirkung entfalten können? Doch mir geht es nur um die Frage, wie es zu dem anfänglichen Scherbenhaufen kam.

I.

Weshalb hat sich Adenauer urplötzlich für einen ausgewachsenen Vertrag erwärmt? Ich lasse die lange Vorgeschichte beiseite, die eng mit den nicht vorankommenden Plänen für eine Politische Union der Sechs zusammenhängt. ${ }^{\text {is }}$ Wie wir wissen, brachte de Gaulle bei einem der Gespräche während des triumphalen Staatsbesuchs im September 1962 die Frage nach einem Mechanismus für künftige enge Konsultationen aufs Tapet. ${ }^{16}$ Adenauer jedenfalls zuckte damals zurück, sprach nur von einem vertraulichen "gentlemen's agreement $\ll,{ }^{17}$ doch argumentierte de Gaulle, dieses existiere ja bereits. Der

13 Konrad Adenauer, Teegespräche 1961-1963, bearb. von Hans Peter MEnSING, Berlin 1992, S. 340, Informationsgespräch, 11.3.1963. Streng vertraulich.

14 Ibid. S. 350, Informationsgespräch, 30.5.1963. Streng vertraulich; vgl. auch Horst OSTERHELD, "Ich gehe nicht leichten Herzens...«. Adenauers letzte Kanzlerjahre - ein dokumentarischer Bericht, Mainz 1986, S. 216 (3.6.1963).

is Vgl. u.a. das Themenheft Du Plan Fouchet au Traité franco-allemand de janvier 1963, in: Revue d'Allemagne et des pays de langue allemande, 29 (1997) 2.

16 Vgl. Tischrede de Gaulles auf Schloß Brühl, 4.9.1962, in: Charles DE GaULLE, Discours et Messages, Bd. 4, Paris 1970, S. 4-6

17 Konrad ADENAUER, Erinnerungen 1959-1963. Fragmente, Stuttgart 1968, S. 179. 
General wollte etwas Praktisches tun und dachte an eine Konzertierung der diplomatischen Zusammenarbeit. So ließ sich der Kanzler auf de Gaulles Idee eines Protokolls oder Memorandums einstimmen, wozu dieser einen Entwurf versprach, $^{18}$ den er dann schon relativ rasch nach dem Besuch - am 19. September - als sechs Seiten starken Entwurf eines Protokolls über die organisierte Zusammenarbeit in den Bereichen Außenpolitik, Verteidigung, Jugend und Erziehung übersandte. ${ }^{19}$ In den folgenden Monaten wurde dieser Text zwar verschiedentlich verändert, ohne daß aber das Prinzip eines Protokolls irgendwie in Frage gestellt worden wäre. Nun ist schon damals und später von den Historikern vermutet worden, daß Adenauer durchaus zu weitergehenden Vereinbarungen bereit war, sich aber mit Blick auf die ihm bestens bekannte innenpolitische Szenerie, doch genauso auch mit Blick auf die USA, England und die Sechser-Gemeinschaft vorerst bedeckt hielt. Bekanntlich liegt den Entscheidungen von Spitzenpolitikern zumeist ein ganzes Bündel von Motiven zugrunde. Das gilt ganz besonders für einen alten Fuchs wie Adenauer. Die wichtigsten dieser eng verschlungenen Motive, die aus seiner Sicht für einen privilegierten, aber dennoch nicht vorbehaltlosen deutsch-französischen Bilateralismus sprachen, waren:

a. Die von Beginn der Kanzlerschaft, also seit 1949, spürbare Aufgeschlossenheit für enges, auch bilaterales Zusammengehen der einstigen »Erbfeinde ${ }^{20}{ }^{20}$ Diese mündete allerdings schon mit dem Schuman-Plan vom Mai 1950 und den folgenden Integrationsplänen in den Multilaterialismus der SechserGemeinschaften ein, überwölbt von der unbedingten Priorität der Beziehungen zu Washington. Dennoch, eine latente Disposition zum deutsch-französischen Bilateralismus war immer vorhanden und gegebenenfalls aktivierbar.

b. Die Fixierung auf Amerika schwächte sich zwischen 1959 und $1962 \mathrm{zu}$ sehends $a b,{ }^{21}$ als schon die Eisenhower-Administration und danach erst recht die Kennedy-Administration auf den scharfen Druck Chruschtschows gegen die westliche Berlin-Präsenz und die Grundsatzpositionen in der deutschen Frage so reagierten, wie es der gesunde Menschenverstand gebot: Notfalls lieber Kompromisse auf Kosten der Bundesrepublik als ein Krieg, und wenn schon ein Krieg, dann lieber noch ein kurzer konventioneller Krieg in Deutschland als ein umfassender Atomkrieg. England unter Premierminister

18 Vgl. LAPPENKÜPER (Anm. 3), S. 1744.

$19 \mathrm{Vgl}$. Archives nationales, 5 AG 1/165, de Gaulle an Adenauer, 20.9.1962, Très secret; ibid. Memorandum, 19.9.1962; MAE/Paris, EU 1961-1970, RFA, Bd. 1574/1598.

20 Vgl. Horst MÖLLER, Konrad Adenauers Frankreichpolitik bis zum Amtsantritt Charles de Gaulles, in: Klaus MANFRASS (Hg.), Paris - Bonn. Eine dauerhafte Bindung schwieriger Partner, Sigmaringen 1984, S. 170-178.

21 „Überhaupt, der Kanzler geht auf engste Partnerschaft mit Frankreich aus«, notiert der CDU-Fraktionsvorsitzende Heinrich Krone in sein Tagebuch am 3. März 1959; Heinrich KRONE, Tagebücher. Erster Band: 1945-1961, bearbeitet von Hans-Otto KLEINMANN, Düsseldorf 1995, S. 337. 
Harold Macmillan ging zeitweise am weitesten. Die dramatischste Abkühlung im Verhältnis zu Washington erfolgte Mitte April 1962, als Washington ein sehr weitgehendes amerikanisches Berlin-Papier ultimativ übersandte, das wohl auf Veranlassung Adenauers - durch ein "Leck" der deutschen Presse zur Abschlachtung überantwortet wurde. ${ }^{22}$ De Gaulle verhielt sich während der ganzen Krisenjahre vorbildlich, was ihn freilich nicht viel kostete, Adenauer aber half, durch Verzögerungsmanöver über die Runden zu kommen. Es ist jedenfalls auffällig, daß die seit Mai 1962 bei Adenauer sichtlich aufblühende Sympathie für eine Zweier-Politik im Bund mit Frankreich nicht nur mit der schmeichelhaften Einladung zu einem großen Staatsbesuch koinzidierte, sondern auch mit seinem tiefen Groll gegenüber den schwankenden Amerikanern und auch den Briten.

c. Ernst zu nehmen sind die Hinweise, daß die Intensivierung des deutschfranzösischen Bilateralismus auch taktisch bedingt war, um vor allem in der Nuklearfrage Druck auf die USA auszuüben. ${ }^{23}$ Ich nenne auch hier nur eine einzige Koinzidenz. Am 14. Januar 1963, als sich Adenauer insgeheim schon entschieden hatte, für die Vereinbarung mit Paris die Vertragsform vorzuschlagen, kam US-Unterstaatssekretär George Ball nach Bonn, um mit Adenauer und dem neuen Verteidigungsminister Kai-Uwe von Hassel über das Projekt der Multilateral Force (MLF) zu sprechen. Beide versicherten ihm, die Bundesrepublik werde sich mit allen Kräften an diesem Projekt beteiligen. ${ }^{24}$ $\mathrm{Zu}$ diesem Zeitpunkt war dem Kanzler aber bereits völlig klar, daß de Gaulle es ablehnte, seine Force de frappe vom Netz eines NATO-Konzepts einfangen zu lassen. Was Adenauer damals, unterstützt vom Auswärtigen Amt und vom Verteidigungsministerium, nuklear noch alles wünschte, kam in dieser Unterredung zur Sprache: weitere atomare Gefechtsfeldwaffen für die Bundeswehr, gleichberechtigte Mitsprache beim Kernwaffeneinsatz, eine NATO-Atommacht und mobile Mittelstreckenraketen in Europa, ${ }^{25}$ die jedoch erst 20 Jahre später, im Jahre 1983 kamen. Es ist nicht auszuschließen, daß die Intensivierung der Verteidigungskooperation mit de Gaulle auch als Wink mit dem Zaunpfahl an die amerikanische Adresse gerichtet war. Erinnert sei aber auch daran, da $B$ Adenauer gerne zweigleisig fuhr. Warum also, so vielleicht die Überlegung, nicht ausloten, ob die Bundesrepublik nicht im Zusammenwirken mit Frankreich den Finger an den atomaren Drücker bekommen konnte? Jedenfalls beweist das "Ja« zur MLF, wie wenig der Kanzler an eine einseitige Ankoppelung der Verteidigungspolitik an Paris dachte. Angesichts der Kräfte-

Vgl. Aufzeichnung Adenauers vom 20.4.1962; Stiftung Bundeskanzler-Adenauer-Haus Rhöndorf [StBKAH] III 52. Streng geheim.

23 $\mathrm{Vgl}$. Eckart CONZE, Die gaullistische Herausforderung. Die deutsch-französischen Beziehungen in der amerikanischen Europapolitik 1958-1963, München 1995.

24 SCHWARZ (Anm. 8), S. 812.

25 Vgl. Unterredung Adenauers mit de Gaulle, 22.1.1963; StBKAH III 62. Geheim. 
verhältnisse im Kabinett und in der Koalition hätte er dies auch gar nicht gekonnt, und jeder Blick auf die Machtverhältnisse im Ost-West-Verhältnis hätte dies ohnehin zur Verrücktheit gestempelt.

d. Erwähnt sei hier nur die alte Sorge vor einem Wiederaufleben der französisch-russischen Allianz, auf die er gerade in diesen Wochen immer wieder zu sprechen kam. Nicht nur um Schröder für den deutsch-französischen Vertrag zu gewinnen, erinnert er ihn an den französischen Flottenbesuch in Kronstadt 1891, an den Staatsbesuch Raymond Poincarés in St. Petersburg im Juli 1914, an den Ersten Weltkrieg und an den französischen-sowjetischen Vertrag von 1944. Seine Angst vor einer Einkreisung ist reell. ${ }^{20} \mathrm{DaB}$ diese nicht ganz unbegründet war, zeigte die dramatische Annäherung de Gaulles an die Sowjetunion im Zeichen ost-westlicher Entspannung sowie der Ablehnung amerikanischer Hegemonie zwischen 1966 und $1968 .^{27}$

e. Schließlich ein entscheidendes Motiv, vielleicht das in jenen Monaten wichtigste: Adenauer schätzte in de Gaulle einen Staatsmann ersten Rangs, der mit ihm zusammen weitreichende Gestaltungspläne auf den Weg bringen möchte. Im Vergleich mit de Gaulle fielen die anderen damaligen Granden des Westens ab: der schwankende, als Showman mehr als durch Substanz überzeugende Kennedy oder der fischige, spürbar germanophobe Macmillan, die allesamt nur über die Runden kommen wollten. $\mathrm{Da}$ de Gaulle auch ihm selbst, Adenauer, den allergrößten Respekt zollte, mag ihn emotional nicht ganz gleichgültig gelassen haben. Je älter und je innenpolitisch angeschlagener er sich fühlte, um so offener war er somit für die Zusammenarbeit mit de Gaulle, obgleich ihm die politische und persönliche Problematik dieser Verbindung durchaus bewußt war. Denn natürlich kannte Adenauer alle Argumente, die gegen einen zu engen oder gar exklusiven deutsch-französischen Zweibund sprachen, und er hat diese oft selber formuliert: Nein, so hörte Botschafter Herbert Blankenhorn im Februar 1961 von ihm, de Gaulle dürfe aus der Bundesrepublik keinen »französischen Satellitenstaat « machen; ${ }^{28}$ nein, so erklärte er wieder und wieder, wenn nur alle Sechs in der EWG zu einer Politischen Union bereit wären, dann bedürfte es keiner Zweier-Union mit Frankreich - 1962 wußte er allerdings genau, daß eine Politische Union zu Sechs nicht mehr in den Karten war, auch de Gaulle war jetzt dagegen -; nein, so versicherte er Walter Hallstein, und Heinrich von Brentano und den Partnern in Italien und in den Benelux-Ländern, die integrierte EWG müsse und werde natürlich weiterentwickelt werden; eben dafür auch den primär auf intergouvernementale Zusammenarbeit abonnierten de Gaulle zu gewinnen, sei gera-

$26 \mathrm{Vgl}$. SCHWARZ (Anm. 8), S. 815.

27 Vgl. Knut LiNSEL, Charles de Gaulle und Deutschland 1914-1969, Sigmaringen 1998, S. 227ff.; Maurice VAïSSE, La grandeur. Politique étrangère du général de Gaulle 19581969, Paris 1998, S. 413ff.

${ }^{28}$ BAK, NL Blankenhom, Bd. 110, Bl. 211f., Tb. 6.2.1961. 
dezu ein Hauptgrund für die deutsch-französische Entente; nein, man dürfe um Gottes willen auch Amerika nicht entfremden und die NATO nicht aufs Spiel setzen, doch auch in dieser Hinsicht könne es nur von Nutzen sein, de Gaulle in engem deutsch-französischem Zusammenwirken vom Verlassen der atlantischen Allianz abzuhalten.

Das alles wußte er durchaus und sagte es häufig. Dennoch gewinnt man bei gründlicher Analyse seines taktischen Hakenschlagens doch den Eindruck, daß die Argumente für eine deutsch-französische Zweier-Union - in welchen Formen auch immer - seit Frühjahr 1962 zusehends stärker wogen als die Bedenken. Aber noch einmal: Warum ist er nicht bei dem viel risikoloseren Konzept eines Protokolls verblieben? Schließlich wollte ja nicht einmal de Gaulle, der damals eher der Drängende war, viel weiter gehen. Denn 1962 und 1963 waren nicht nur die wesentlichen Partner-Regierungen gegen jeden Ansatz eines deutsch-französischen Zweibundes, sondern auch ein überwiegender Teil der bundesdeutschen Öffentlichkeit. ${ }^{29}$ Etwas überspitzt formuliert, lauteten die seinerzeitigen außenpolitischen Prioritäten bei großen Teilen der seinerzeitigen politischen Klasse in Bonn, bei der Presse und auch in der Wirtschaft: 1. Amerika; 2. Vollendung der EWG und baldige Erweiterung um England; 3. Frankreich. In der CDU waren die Schwergewichte im Kabinett, die Kanzlerprätendenten Erhard und Schröder sichtlich frankophob, genauer gesagt: anti-gaullistisch, der Fraktionsvorsitzende von Brentano und große Teile der CDU/CSU-Fraktion genauso. Dasselbe galt für die FDP. Auch die SPD-Opposition war in jenen Jahren eben dabei, sich nach dem Wahlsieg Kennedys zur 150prozentig pro-amerikanischen Partei zu mausern, während de Gaulle weithin in der Partei immer noch als halb-faschistischer Diktator verdächtigt wurde. ${ }^{30}$

Von den Schwergewichten im Kabinett unterstützte nur der zunehmend Amerika-kritische Verteidigungsminister und CSU-Vorsitzende Franz Josef Strauß den profranzösischen Kurs Adenauers, doch er brachte sich selbst durch die »Spiegel-Affäre ${ }^{31}$ vom Oktober/November 1962 vorerst um jeden politischen Einfluß. Adenauer, der ohnehin kaum hoffen durfte, als Bundeskanzler das Jahr $1963 \mathrm{zu}$ überleben, fand sich also bei seinem offenkundigen

29

Vgl. François-Georges DREYFuS, L'opinion allemande devant le traité de l'Élysée du 22 janvier 1963, in: Relations internationales, (1984) 37, S. 111-119; DERS., L'opinion française et le traité de l'Élysée, in: Revue d'Allemagne et des pays de langue allemande, 29 (1997) 2, S. 201-209.

$30 \mathrm{Vgl}$. Günter BUCHSTAB, Zwischen "Zauber und Donner«. Die CDU/CSU und de Gaulle, in: Wilfried LOTH, Robert PICHT (Hg.), De Gaulle, Deutschland und Europa, Opladen 1991, S. 95-108; Beatrix W. BOUVIER, Die SPD und Charles de Gaulle in den sechziger Jahren, in: ibid. S. 109-120; Reiner MARCOWITZ, Option für Paris? Unionsparteien, SPD und Charles de Gaulle 1958-1969, München 1996.

"Vgl. Dorothee LIEHR, Von der Aktion gegen den Spiegel zur Spiegel-Affäre. Zur gesellschaftspolitischen Rolle der Intellektuellen, Frankfurt a. M. 2002. 
Kurswechsel in Richtung Paris innenpolitisch ziemlich allein, er wußte dies natürlich auch, und genauso wußte man dies in Paris. Umso schwerer zu beantworten ist also die Frage: Warum um alles in der Welt schaltete Adenauer ausgerechnet nach der weiteren Schwächung durch die "Spiegel-Affäre« in den beiden ersten Januarwochen abrupt auf einen Vertrag um?

Die schlichte Antwort kann niemanden befriedigen, der bei großen Staatsmännern stets weitreichende, gut überlegte Strategien vermutet: Erst Anfang Januar konfrontierte ihn die Rechtsabteilung im Auswärtigen Amt mit der peinlichen Tatsache, daß das Thema "Kultur», das nach Meinung de Gaulles und Adenauers unbedingt von den Konsultationen erfaßt werden sollte, gemäß Art. 59 GG eindeutig Ländersache ist. Groß sei die Gefahr, so das schwer zu widerlegende Argument, da $\beta$ ein Land deshalb vor das Bundesverfassungsgericht ziehe und obsiege. ${ }^{32}$ Welche Peinlichkeit für die deutsch-französischen Beziehungen, auch: welche Peinlichkeit für den Kanzler, der auf diesen entscheidenden Umstand bisher nicht hingewiesen hatte! Eine Peinlichkeit übrigens auch für den auf seine Rechtskenntnisse so stolzen Bundesaußenminister Schröder, der zusammen mit Außenminister Maurice Couve de Murville dem Protokoll persönlich bei einem Treffen in Paris im Dezember 1962 den letzten Schliff gegeben hatte. ${ }^{33}$ Der letztere Umstand erklärt übrigens auch besser als vieles andere, weshalb es Adenauer nicht allzu schwer fiel, Schröder für die Vertragsform zu gewinnen: Dieser wollte sich vor Couve de Murville, doch auch in der deutschen Öffentlichkeit nicht blamieren, indem er eine neue Verhandlungsrunde forderte, wodurch der Unterzeichnungstermin am 22. Januar 1963 hinfällig geworden wäre. Da eine Verschiebung inopportun erschien, blieb nach Meinung Adenauers, aber auch Schröders nur die blitzschnelle Emporstufung des Protokolls in einen Vertrag. Daß ein langgezogener Ratifikationsproze $B$ alle latenten Gegner der Annäherung an Frankreich auf den Plan rufen und ihnen ein hervorragendes Marterwerkzeug in die Hand drücken würde, wurde nicht genau bedacht. Und wenn, so mochte sich Adenauer sagen, daß er über große Erfahrung und viel Chuzpe verfügte, innenpolitisch höchst umstrittene internationale Verträge durchzusetzen. Ob ihn in den entscheidenden Tagen nun auch die Überlegung bewegte, die privilegierten Beziehungen mit Frankreich durch einen feierlich ratifizierten Vertrag weit über seine Amtszeit hinaus zu sichern, ist zwar bald behauptet worden; sichere Zeugnisse dafür existieren aber nicht. Viel wahrscheinlicher ist, da $\beta$ er am 21. Januar 1963 nicht mit leeren Händen nach Paris kommen wollte.

32 Vgl. LAPPENKÜPER (Anm. 3), S. $1767 \mathrm{f}$.

33 Vgl. SCHWARZ (Anm. 8), S. 817. 
II.

Vielleicht wäre er tatsächlich relativ unverbellt durchgekommen, hätte de Gaulle nicht mit der Pressekonferenz vom 14. Januar 1963 alle Gegner seiner England- und NATO-Politik in der ganzen westlichen Welt und vor allem in der Bundesrepublik Deutschland aus ihren Löchern herausgelockt. Hatte ihn denn gar niemand unter Bezugnahme auf den Besuch der großen deutschen Delegation am 21./22. Januar gewarnt?

Man muß aber bezüglich de Gaulles für mildernde Umstände plädieren. Drei Wochen lang hatte er die Pressekonferenz sorgfältigst vorbereitet. ${ }^{34}$ In Bezug auf die deutsch-französischen Beziehungen konnte er immer noch von einem fest vereinbarten, nicht ratifikationsbedürftigen Protokoll ausgehen, denn am 14. Januar wußte er überhaupt noch nicht, daß Adenauer im Begriff war, auf einen ratifikationsbedürftigen Vertrag umzuschalten. ${ }^{35}$ De Gaulle hat wahrscheinlich vorausgesehen, daß er durch die Pressekonferenz England und die USA skandalisieren würde. Vor allem die Ablehnung der Nuklearstrategie Kennedys war recht eigentlich beabsichtigt. Nicht erkennen konnte er aber damals, daß er damit dem Gedanken eines sehr engen Bundes mit der Bundesrepublik ungewollt den Todesstoß versetzte. Eine entsprechende Mitteilung der Bonner Botschaft, Bonn wolle unerwarteter Weise einen Vertrag vorschlagen, erreichte ihn erst am Abend des 18. Januar, nachdem Adenauer erst AuBenminister Schröder, dann - am 16. Januar - mit Schröders tätiger Hilfe das Bundeskabinett und am 18. Januar auch die Vorsitzenden aller Bundestagsparteien überzeugt, oder sagen wir besser überrollt hatte. ${ }^{36}$

Im Kreis der Fraktionsvorsitzenden gingen die Wogen schon ziemlich hoch, denn de Gaulles kaum verhülltes, wenngleich noch nicht definitiv präzisiertes "Nein« zum englischen Beitritt erschien als ganz unerträgliche Provokation. Doch Adenauer salvierte sich mit dem Versprechen, auf de Gaulle einzuwirken, die Verhandlungen mit England weiterzuführen. ${ }^{37}$ Aus den Gesprächsprotokollen vom 22 . Januar hat man allerdings erfahren, daß er sich bei diesem nicht stark ins Zeug legte, sondern nur eine gesichtswahrende neue Verhandlung am 29. Januar erbat und auch eine entsprechende Zusage erhielt. ${ }^{38}$ Wenn de Gaulle die rasch auftretenden Schwierigkeiten bei der Ratifikation

34 Vgl. Alain Peyrefitte, C'était de Gaulle, Bd. I: »La France redevient la France«, Paris 1994, S. 349f.

35 OSTERHELD (Anm. 14), S. 170.

$36 \mathrm{Vgl}$. SCHWARZ (Anm. 8), S. 819f.

$37 \mathrm{Vgl}$. OSTERHELD (Anm. 14), S. 185.

38 Unterredung zwischen Bundeskanzler Adenauer und Staatspräsident de Gaulle vom 22.1.1963, Aufzeichnung 115-A.14/63, 25.1.1963. Geheim, in: AAPD 1963, Bd. 1, S. $43 \mathrm{ff}$. 
des Élysée-Vertrages doch auch in starkem Maß selbst verschuldet hat, so deshalb, weil er diese Verhandlung definitiv platzen ließ. Leider, so erklärte Adenauer daraufhin, habe er sich mit seinen Bemühungen letztlich doch nicht durchsetzen können. ${ }^{39}$ Man versteht, weshalb der damalige Finanzminister Adenauers, der FDP-Politiker Heinz Starke, die Verhandlungskünste des Kanzlers im FDP-Parteivorstand am 22. Januar mit den Worten kommentierte: »Er lädt Sie ein, um Sie zu betrügen. Er liest Ihnen etwas vor, und behauptet nachher, Sie hätten zugestimmt. Das ist lebensgefährlich! ${ }^{40}$ Eigentlich hätten nach der Pressekonferenz am 14. Januar bezüglich der Vertragsform bei Adenauer alle Alarmglocken erschallen müssen. $\mathrm{DaB}$ die atlantisch orientierten, pro-britischen und vor allem auf Erweiterung der EWG fixierten Gegner einer zu engen Bindung mit Frankreich ihn jetzt aufgrund der Ratifikationsbedürftigkeit eines Vertrages in der Hand hatten, ist im nachhinein klar. Ob er selbst sich der zu erwartenden Schwierigkeiten voll bewuBt gewesen ist, ist nicht mehr feststellbar. Immerhin konnte er darauf vertrauen, daß selbst die AntiGaullisten im eigenen Kabinett und im Deutschen Bundestag einen Freundschaftsvertrag mit Frankreich schwerlich ablehnen konnten. Auch in diesem Fall kamen die wohlbekannten Charakterzüge Adenauers ins Spiel. In unerwartet kritischen Lagen pflegte er sich häufig rasch festzulegen, dabei bestrebt, ein fait accompli zu schaffen, immer in der meist nicht unbegründeten Erwartung, dank überlegener Willenskraft und mit taktischer Gerissenheit alle Widerstrebenden irgendwie zu überspielen oder sie kleinzukriegen. Der CDUFraktionsvorsitzende Heinrich Krone, der ihn am besten kannte, seufzte in seinem Tagebuch immer wieder einmal: "Es ist toll, wie der Kanzler mit seinen Ministem umspringt. ${ }^{4 !}$ Tatsache ist jedenfalls, daß erst de Gaulles definitives »Nein« zum britischen Beitritt dem Faß den Boden ausgeschlagen hat.

III.

So kam es fast zwangsläufig zur Präambel. Diese stufte den von de Gaulle erstrebten, hoch problematischen deutsch-französischen Zweibund auf das erträglichere und - wie sich später zeigte - durchaus sehr nützliche Niveau eines privilegierten Konsultationsvertrages herab. Ein Liegenlassen des

39 Vgl. LAPPENKÜPER (Anm. 3), S. 1789ff.

40 22.2.1963: Sitzung des FDP-Bundesvorstandes, in: FDP-Bundesvorstand. Die Liberalen unter dem Vorsitz von Erich Mende. Sitzungsprotokolle 1960-1967, bearb. von Reinhard SCHIFFERS, Düsseldorf 1993, S. 480.

41 Heinrich KRONE, Tagebücher. Erster Band: 1945-1961, bearb. von Hans-Otto KLEINMANN, Düsseldorf 1995, S. 433 (14.7.1960). 
Freundschaftsvertrages mit Frankreich oder gar eine Ablehnung wäre nach Lage der Dinge unmöglich gewesen. Seitens der deutschen Betreiber der Präambel bei der FDP, auf die es vor allem ankam, verschaffte man sich mit dem Hinweis ein gutes Gewissen, schließlich sei es in Frankreich eine alte und gängige Gesetzgebungspraxis, in einer Präambel festzustellen: »In der und der und der Erwägung stimmen wir der Ratifizierung des Vertrages zu. ${ }^{42}$ Und natürlich suchte Adenauer - über Alain Peyrefitte - bei de Gaulle den Eindruck zu erwecken, als besage die Präambel überhaupt nichts. ${ }^{43} \mathrm{Da}$ sich de Gaulle aber geprellt vorkommen mußte, versteht sich. Nach deutscher Parlamentspraxis sind eben derart einseitige Präambeln völlig unüblich. Die Tatsache, daß sich die zahlreichen Gegner des Vertrages nicht - wie neun Jahre später beim Moskauer Vertrag - mit einer interpretativen Bundestagsresolution zufrieden gaben, sondern Wert darauf legten, den Élysée-Vertrag mit dem allerschärfsten Skalpell einer zudem noch provozierend ausführlichen Präambel zum Ratifikationsgesetz politisch zu kastrieren, sagte alles über die damalige Erbitterung der Bonner politischen Klasse, aber ebenso über die seinerzeitigen Machtverhältnisse aus. $\mathrm{Zu}$ allem hin wurde im Verlauf des Ratifikationsverfahrens auch noch deutlich, daß Ludwig Erhard das Rennen um die Nachfolge Adenauers gewonnen hatte. Erhard, der jetzt offen auf Gegenkurs ging, sich allerdings nicht $\mathrm{zu}$ einem konstruktiven Mißtrauensvotum verlocken ließ, wohl aber zu einem sehr kritischen, vieldiskutierten Interview mit Ulrich Kempski von der Süddeutschen Zeitung, das den - wie sich zeigen sollte durchaus zutreffenden Satz enthielt: "Der Bilateralismus ist tot. ${ }^{44}$

In der Tat war das Konzept eines exklusiven deutsch-französischen Zweibundes eine Totgeburt. Das Interview zusammen mit weiteren Stellungnahmen Erhards nahm bereits vorweg, was dann drei Jahre lang ablief und de Gaulle zutiefst entfremdete. Auf kurze Sicht fuihrte somit das improvisierte Vertragskonzept in der Bundesrepublik zum Triumph des Atlantizismus. Das eigentliche Wunder aber ist, wie gesagt, darin zu sehen, daß aus dieser Totgeburt eine Art Lebewesen wurde, ein in der neueren Diplomatiegeschichte sehr einzigartiges Lebewesen, dessen 40. Geburtstag wir 2003 begehen.

42 22.2.1963: Sitzung des FDP-Bundesvorstandes, in: FDP-Bundesvorstand. Die Liberalen unter dem Vorsitz von Erich Mende. Sitzungsprotokolle 1960-1967, bearb. von Reinhard SCHIFFERS, Düsseldorf 1993, S. 485.

43 Vgl. Alain PEYREFITTE, C'était de Gaulle, Bd. I: „La France reprend sa place dans le monde «, Paris 1997, S. 237.

44 Vgl. Süddeutsche Zeitung, 5.2.1963. 